\title{
La enseñanza de la matemática y su incidencia en la calidad de graduados en la educación superior
}

\section{The teaching of mathematics and its incidence in the quality of graduates in higher education}

\section{La enseñanza de las matemáticas.}

\author{
Fabrina Monserrate Cedeño Mendoza, MSc. ${ }^{(1)}$ \\ Gilberto Antonio Jarre Cedeño, MSc. ${ }^{(2)}$ \\ Raquel Inés Macías Cedeño, MSc. ${ }^{(3)}$ \\ Gilberto Rafael Jarre Vera MSc. ${ }^{(4)}$ \\ (1) Universidad Técnica de Manabí, Instituto de Ciencias Básicas, Portoviejo - Manabí - Ecuador \\ fmcedeno@utm.edu.ec ORCID: https://orcid.org/0000-0002-6829-6683 \\ (2) Universidad Técnica de Manabí, Facultad de Ingeniería Agrícola, Portoviejo - Manabí - Ecuador \\ gjarre@utm.edu.ec ORCID: https://orcid.org/0000-0003-1087-5938 \\ (3) Unidad Educativa Sucre, 24 de Mayo, Manabí, Ecuador, raquelmacias1998@gmail.com ORCID: \\ https://orcid.org/0000-0002-7644-5829 \\ (4) Universidad Técnica de Manabí, Facultad de Matemáticas, Físicas y Químicas, Portoviejo - Manabí - Ecuador \\ grjarre@utm.edu.ec ORCID: https://orcid.org/0000-0001-9915-0823
}

Contacto: fmcedeno@utm.edu.ec

Recibido: 18-02-2020 Aprobado: 01-04-2020

\section{Resumen}

La presente investigación plantea el problema de las insuficiencias didácticas en la enseñanza de Matemática y la calidad de graduados en la educación superior; para lo cual se estableció los objetivos: a) Caracterizar el proceso de monitoreo que ejercen las autoridades en los docentes en el desarrollo del microcurrículo para establecer el cumplimiento de los syllabus, b) Evaluar la enseñanza que se les brinda a los estudiantes en el dominio de conocimientos matemáticos, c) Determinar el involucramiento del área de ciencias exactas en la didáctica Matemática en el proceso pedagógico. Es preciso partir, en el análisis específico de la enseñanza y el aprendizaje de la Matemática, del generalizado rechazo y temor hacia ellas existente en nuestra sociedad. Será necesario superar este obstáculo, pero existe otra serie de dificultades adicionales que es necesario reconocer. El tratamiento metodológico de la investigación está basado en las siguientes modalidades: bibliográfica, de Campo, y Cuasi- experimental. Además, se usó los siguientes tipos de investigación: exploratorio, el descriptivo, el analítico, el sintético, el propositivo el cual permitió a los investigadores, plantear una propuesta que conlleve a solucionar la problemática investigada. Los resultados indican que los docentes si aplican técnicas en el proceso pedagógico intra-aula, las mismas que facilitan el aprendizaje significativo de los estudiantes garantizando la calidad de graduados, aseverando como conclusión que los docentes y estudiantes si llevan a cabo el proceso de evaluación de aprendizajes.

Palabras Claves: Enseñanza, matemática, educación, superior.

\section{Sumary}

The present investigation raises the problem of inadequacies didactics in the teaching of Mathematics and the quality of graduates in education higher; for which the objectives were established: a) Characterize the monitoring process that the authorities exercise in teachers in the development of the micro-curriculum for establish compliance with the syllabus, b) Evaluate the teaching provided to students in the domain of knowledge mathematicians, c) Determine the involvement of the area of exact sciences in the 
Mathematical teaching in the pedagogical process. It is necessary to start, in the analysis specific to the teaching and learning of Mathematics, of widespread rejection and fear towards them existing in our society. It will be necessary to overcome this obstacle, but there is another series of additional difficulties that need to be recognized. In general, students instead of being attentive to reasoning and participating in class, are limited, by tradition of learning, to take notes that will then try to Memorize when studying for your exams. The methodological treatment of the research is based on the following modalities: bibliographic, Field, and Quasi-experimental. In addition, the following types of research: exploratory, descriptive, analytical, synthetic, the purpose which allowed the researchers to propose a proposal that entails to solve the problem investigated. The results indicate that teachers do apply techniques in the intra-classroom pedagogical process, which facilitate the meaningful student learning guaranteeing the quality of graduates, asserting as a conclusion that teachers and students do carry out the learning evaluation process.

Keywords: Teaching, mathematics, education, higher.

\section{Introducción}

La mayor parte de los maestros de Matemática, se han formado en escuelas o facultades de Matemática en donde la interacción con otras disciplinas, inclusive tan cercanas como la física, es tradicionalmente escasa.

La matemática ha sido conceptualizada como la ciencia de los números y las formas, las relaciones y las medidas, las inferencias y sus características apuntan hacia la precisión, el rigor, la exactitud (Cantoral, 1995). Por consiguiente, la matemática está asociada a valores dominantes en la vida social y privada corrientes de la llamada sociedad moderna.

Los grandes héroes de la matemática, esto es, aquellos autores responsables del avance y consolidación de esa ciencia son identificados en la antigüedad griega y, posteriormente, en la Edad Moderna en los países centrales de Europa, sobre todo Inglaterra, Francia, Italia, Alemania. Los nombres más recordados son Tales, Pitágoras, Euclides, Descartes, Galileo, Newton, Leibniz, Gauss, Hilbert. Son ideas y hombres originarios de Europa, del norte del Mediterráneo, que se impusieron a las ideas y prácticas de las poblaciones indígenas que fueron dominadas en el proceso de conquista y colonización (Rafael, 1993).

Durante las décadas de los años cuarenta y cincuenta así como lo menciona (Artigue, Douady, Moreno, \& Gómez, 1995), se había desarrollado una ingente labor de sistematización de matemática a través del lenguaje de la teoría de conjuntos y de la lógica matemática, liderada por el grupo que escribía con el seudónimo de "Nicolás Bourbaki". Esta reestructuración bourbakista de las matemáticas sedujo a la comunidad matemática por su elegancia arquitectónica y por la unificación del lenguaje, hasta tal punto que se pensó abolir el plural "matemáticas" para hablar de una sola "matemática".

La educación superior siempre ha sido considerada como el motor fundamental para el desarrollo de los pueblos, esta permite a los seres humanos acrecentar sus valores y cualidades, ayudándolo a formar su personalidad y convertirlo en una persona capaz de satisfacer sus necesidades personales, servir a su familia, a la sociedad y al Estado.

En su afán por ofrecer nuevas oportunidades a sus graduados y profesionales del país debido a los altos niveles de competitividad producto de un Ecuador globalizado, resulta necesario para los docentes adaptarse a nuevos cambios y exigencias de los mercados actuales, con la finalidad de promover la actualización académica, la insuficiencia didáctica en la enseñanza de matemática, desarrollo de habilidades y aptitudes hacia el fortalecimiento de conocimientos y complementar su formación profesional con una metodología formal y aplicada.

En nuestro sistema educativo, la enseñanza verbalista tiene una larga tradición y los alumnos están acostumbrados a ella, esta poderosa inercia ha impedido a los estudiantes percatarse que, en las ciencias, en particular en la matemática, lo importante es entender. Es preciso partir, en el análisis específico de la enseñanza y el aprendizaje de la matemática, como lo menciona (Beltrán, 1996) del generalizado rechazo y temor hacia ellas existente en nuestra sociedad. Será necesario superar este obstáculo, pero existe otra serie de dificultades adicionales que es necesario reconocer.

En lo general, los estudiantes en lugar de estar atentos a los razonamientos y participar en clase, se limitan, por tradición de aprendizaje, a tomar apuntes que después tratarán de memorizar al estudiar para sus exámenes (Luis, 2013). Un gran número de factores contribuyen a que esta situación no cambie: con frecuencia el maestro está acostumbrado a este estado de cosas y lo ve como natural; por lo extenso de los programas, el maestro decide cubrirlos en su totalidad y no se da tiempo para generar el diálogo, fomentar las intervenciones de los estudiantes y hacerles ver que es posible sacar más provecho a los tiempos de las clases. 
Es útil mencionar que los tipos de aprendizaje memorístico y significativo son los extremos de un continuo en el que ambos coexisten en mayor o menor grado y en la realidad no podemos hacerlos excluyentes (Granados \& García, 2016). Muchas veces aprendemos algo en forma memorista y tiempo después, gracias a una lectura o una explicación, aquello cobra significado para nosotros; o lo contrario, podemos comprender en términos generales el significado de un concepto, pero no somos capaces de recordar su definición o su clasificación.

Esta investigación se desarrolló con los siguientes objetivos: a) Caracterizar el proceso de monitoreo que ejercen las autoridades en los docentes en el desarrollo del micro-currículo para establecer el cumplimiento de los syllabus, b) Investigar la aplicación de las técnicas activas para la enseñanza de Matemática en el proceso pedagógico intra-aula, c) Evaluar la enseñanza que se les brinda a los estudiantes en el dominio de conocimientos matemáticos, d) Determinar el involucramiento del área de ciencias exactas en la didáctica Matemática en el proceso pedagógico.

La contribución que brindó esta investigación es el de mejorar la calidad de los graduados con respecto a las insuficiencias didácticas en la enseñanza de matemática en la educación superior.

\section{Materiales y métodos}

El trabajo de investigación científica se apoyó en el método bibliográfico o de recopilación teórica porque se indagó como sustentación las informaciones relacionadas para las variables dependientes e independientes en fuentes como el internet, textos, acuerdos internacionales y los proyectos de similar contenido que existen en las bibliotecas de los centros de educación superior, de igual forma se empleó la metodología de campo porque esta se constituye en la aplicación práctica de la teoría para comprobar si ella funciona o no en la propuesta objeto de investigación mediante encuestas, entrevistas y guías de observaciones. Así mismo la población fue de 105 encuestados entre estudiantes y docentes; el instrumento de encuesta está basado en un total de 9 preguntas para docentes y 9 preguntas para estudiantes con 4 ítems como respuesta. Con la aplicación del método exploratorio que se constituye en el nivel más elemental de la investigación, se recopilaron los primeros datos del objeto de la investigación. Para analizar los datos estadísticos que se obtuvo del trabajo de campo se utilizó el método analítico que permite explicar e interpretar toda la información cuantitativa.

\section{Resultados}

Con el propósito de investigar con qué frecuencia las autoridades de educación superior realizan el monitoreo del cumplimiento del micro currículo, los docentes encuestados emitieron las siguientes respuestas: el 33,33\% respondió que siempre, el $66,67 \%$ afirma que a veces, las alternativas rara vez y nunca no obtuvieron frecuencia; de tal forma de los estudiantes encuestados se obtuvieron los siguientes resultados: el 20,99\% respondieron siempre, el 65,43\% contestaron que a veces, el 9,88\% afirmaron rara vez, el $3,70 \%$ certificaron que nunca.

$\mathrm{Al}$ respecto sobre la incidencia del monitoreo del micro - currículo en el cumplimiento de los syllabus se logró obtener los siguientes resultados: de los docentes encuestados en su totalidad que corresponde al 100\%, respondieron a la alternativa a) mucho, a diferencia de las respuestas obtenidas por los estudiantes el 40,74\% respondieron que mucho, el $54,32 \%$ afirmaron que poco y el $4,94 \%$ contestaron que nada.

Con la intención de investigar el monitoreo que hacen las autoridades de educación superior hacia los docentes para el cumplimiento de los syllabus, se alcanzaron los siguientes resultados por parte de los docentes: el 33,33\% señala que alto, el $66,67 \%$ respondieron que es bajo; por parte de los estudiantes; el $38,27 \%$ con la alternativa a) alto, el $53,09 \%$ contestaron al literal b) medio y con $8,64 \%$ escogieron el literal c) bajo.

$\mathrm{Al}$ evaluar el dominio de conocimientos matemáticos que poseen los estudiantes; el $66,67 \%$ de los docentes respondieron a la opción alto, y el 33,33\% seleccionando la opción medio; de igual manera los estudiantes encuestados con un porcentaje del $74,07 \%$ respondieron alto y con el 25,93\% afirmaron medio.

Para conocer la frecuencia que el docente evalúa la enseñanza que le brinda a sus estudiantes se obtuvieron los siguientes resultados; el $66.67 \%$ de los docentes escogieron la alternativa a) alto y el $33.33 \%$ la alternativa b) medio; así mismo los estudiantes respondieron con el 65,43\% literal a) siempre, el 32,1\% escogieron el literal b) a veces y con el literal c) rara vez y finalmente la respuesta nunca, no encontró frecuencia alguna.

Además, se investigó si los conocimientos matemáticos que se brinda a los estudiantes ayudan a mejorar la calidad de graduados en la educación superior obteniendo los siguientes resultados: la totalidad de los docentes encuestados que representa el $100 \%$ 
expresaron que sí; y de los estudiantes encuestados el $96,3 \%$ respondieron si y el restante $3,7 \%$ afirmaron no.

La pregunta que indagó sobre el involucramiento del área de ciencias exactas en el proceso pedagógico de la educación superior, obtuvo la siguiente respuesta: de los docentes encuestados el $3,33 \%$ escogió la alternativa a) definitivamente si, y el $66,67 \%$ con la alternativa b) probablemente sí, y de los estudiantes encuestados los resultados se dieron de la siguiente manera: el $35,8 \%$ de los escogieron la alternativa a) definitivamente si, con el $50,62 \%$ eligieron la alternativa b) probablemente sí, y el $13,58 \%$ con la alternativa c) indeciso.

Al analizar si los docentes del área de ciencias exactas desarrollan con eficacia y eficiencia el proceso pedagógico, se lograron los resultados siguientes: los docentes con el 33,33\% respondió que totalmente si, y con el $66,67 \%$ contestaron que parcialmente; los estudiantes respondieron totalmente, que equivale al $43,21 \%, 46$ y el $56,79 \%$ con respuesta parcialmente.

Para investigar con qué frecuencia el área de ciencias exactas se involucra en el proceso pedagógico de la Matemática de los docentes encuestados el 100\% respondieron a la alternativa a) siempre; y de los estudiantes encuestados que constituyen el 55,56\% siempre, el 38,27\% con una respuesta de a veces y el $6,17 \%$ afirmaron que rara vez.

Para entender la labor educativa, es necesario tener en consideración otros tres elementos del proceso educativo: los profesores y su manera de enseñar; la estructura de los conocimientos que conforman el currículo y el modo en que éste se produce y el entramado social en el que se desarrolla el proceso educativo.

Se hace necesario que los docentes del área de ciencias exactas establezcan destrezas para que su labor educativa se realice de acuerdo al proceso educativo que se lleva a cabo con los estudiantes, teniendo una relación directa con el nivel académico, donde un buen ambiente de estudio, la planificación, los métodos utilizados y la motivación por el estudio que impartan los docentes inciden en el aprendizaje del educando.

\section{Discusión}

El monitoreo de las autoridades hacia el trabajo docente es indispensable para el proceso educativo, este permite recoger y registrar información sobre potencialidades, debilidades y necesidades del profesor dentro del micro currículo, para luego ser transformadas en fortalezas que garanticen el desarrollo de competencias en los educandos.

Al respecto (Ausubel 1983), mediante su teoría, enfatiza la importancia que tiene para los estudiantes la formación intelectual. Dicha situación implica para los docentes un reto, ya que supone para ellos la búsqueda de estrategias de enseñanza-aprendizaje así como actividades de aprendizaje y evaluación que conduzcan a interesar a los alumnos en el aprendizaje del contenido de la materia, lo cual a su vez será el medio con el que se buscará desarrollar habilidades y valores implícitos en la disciplina estudiada, así como los requeridos para enfrentar un mundo cada vez más complejo, este autor propugna por el aprendizaje verbal significativo, que permite el dominio de los contenidos curriculares que se imparten en las escuelas, principalmente a nivel medio y superior.

De acuerdo a la psicología cognitiva actual, la tarea del docente debe consistir en programar las actividades o situaciones de aprendizaje adecuadas que permitan conectar activamente la estructura conceptual de una disciplina con la estructura cognoscitiva previa del alumno. Todo proceso educativo, requiere de seguimiento, control, monitoreo, es decir, evaluación, que den a conocer o evidencien los resultados que se van obteniendo, de modo contrario los procesos serán incompletos y se desconocerá sus verdaderos alcances.

Así mismo (Ausubel, 1969) indica "la interrelación del currículo con el profesor y el alumno, es que el currículo es la base para que el proceso de enseñanzaaprendizaje pueda darse de manera organizada y secuencial siempre y cuando el profesor y el estudiante sepan seguirlo y aprovecharlo". Por otro parte el aprendizaje activo, además, puede llegar a suponer un aprendizaje relevante, que produzca en el estudiante la reestructuración de sus esquemas mentales y la adquisición de nuevos y más complejos conocimientos y habilidades, en el que el docente rescata un papel muy importante como organizador y secuenciador de contenidos, con la idea de generar nuevos aprendizajes, como resultado de las nuevas relaciones desencadenadas por la educación superior; es decir, según el tipo de cuestiones que se les plantean a los estudiantes durante una prueba, por ejemplo, estos pueden verse llevado a crear otros puentes cognoscitivos, para resolver esta situación, distintos de lo que había elaborado durante el periodo de enseñanza aprendizaje.

Los estudiantes, como personas, difieren en la cantidad y calidad de información y en el tipo de experiencias previas que poseen, diferencias que influyen en la forma de comprender cada concepto o información. La 
comprensión depende de la competencia que tenga el estudiante para establecer una red de interconexiones entre los conocimientos y experiencias antepuestas con las nuevas ideas que se presentan.

En términos sustantivos, lo que Ausubel está diciendo es que para facilitar el aprendizaje significativo es preciso prestar atención al contenido y a la estructura cognitiva, procurando "manipular" los dos. Es necesario hacer un análisis conceptual del contenido para identificar conceptos, ideas, procedimientos básicos y concentrar en ellos el esfuerzo instruccional. Es importante no sobrecargar al estudiante de informaciones innecesarias, dificultando la organización cognitiva. Es preciso buscar la mejor manera de relacionar, explícitamente, los aspectos más importantes del contenido de la materia de enseñanza con los aspectos específicamente relevantes de la estructura cognitiva del aprendiz.

En tal sentido, atendiendo la concepción constructivista y el aprendizaje significativo de Ausubel "El perfil del docente se orienta a la combinación de elementos importantes que le permiten destacar su labor por la trascendencia y plenitud de su ejercicio, reflejado en la condición de los seres que apoya cada día en su comunidad, así como la contribución de éstos a la toma de decisiones que mejoren su calidad de vida, la de sus semejantes, la naturaleza y el mundo". El problema fundamental se centra en el desconocimiento de cómo se producen los procesos de aprendizaje (articulados entorno a una estructura jerárquica de la mente) y en la aplicación de programas educativos y planes de enseñanza inadecuados, que no respetan los aspectos sustanciales y programáticos del contenido de las asignaturas objeto de estudio por parte de los estudiantes, tendentes a su adquisición y retención significativas.

Dentro del constructivismo se considera al docente como aquel profesional reflexivo, que realiza una labor de mediación entre el conocimiento y el aprendizaje de sus estudiantes, al compartir experiencias y saberes en un proceso de negociación o construcción conjunta del conocimiento y presta una ayuda pedagógica ajustada a la diversidad de necesidades, intereses y situaciones en que se involucran sus estudiantes; es decir, la función central del docente es esencialmente orientar y guiar la actividad mental constructiva, a quienes proporcionará ayuda pedagógica ajustada a su competencia.

\section{Conclusiones}

Se concluye con los resultados estadísticos de la encuesta realizada a los docentes que el proceso de monitoreo que ejercen las autoridades en cuanto al desarrollo del micro-currículo, syllabus, es bajo, el monitoreo de las planificaciones debe ser continua con la finalidad de efectuar correcciones y fortalecer el trabajo docente, en referencia a los resultados de la indagación efectuada a los estudiantes se concluyó que las autoridades de educación superior a veces realizan el monitoreo lo cual coincide con las deducciones ante mencionadas.

Otro punto importante que se obtuvo es la evaluación de los conocimientos matemáticos que dominan los estudiantes concluyendo que estos conocimientos son altos, los mismos que a su vez son evaluados por los docentes mejorando la calidad de graduados en la educación superior, la conclusión que se obtuvo de los estudiantes evidencia lo expuesto por los docentes; las autoridades corroboran este resultado al decir que los docentes y estudiantes si llevan a cabo el proceso de evaluación de aprendizajes.

De la misma manera esta investigación permitió determinar el involucramiento del área de ciencias exactas dando como resultado en la encuesta que esta deberá involucrarse más en la didáctica Matemática del proceso pedagógico, permitiendo que los docentes desarrollen parcialmente los procesos pedagógicos, este mismo criterio es declarado por los estudiantes; las autoridades sin embargo manifiestan que el área si participa de los procesos pedagógicos y que estos son importantes para el desarrollo de competencias.

\section{Bibliografía}

Artigue, M., Douady, R., Moreno, L., \& Gómez, P. (1995). Ingeniería didáctica en educación Matemática. Grupo Editorial Iberoamericana, 97-140.

Ausubel, D. P. (1968). Educational psychology: A cognitive view. New York: Holt, Rinehart and Winston. trad. cast. Psicología educativa: un punto de vista cognoscitivo.

Beltrán, J. (1996). Procesos, estrategias y técnicas de aprendizaje. Revista Complutense de Educación.

Beltrán, M. J. (2011). ¿Qué es el curriculum? Pedagogía Magna.

Cantoral, R. (1995). Matemática Educativa. Revista especializada en educación.

Denyer \& Furnemont. (2004). Las competencias en la educación. Un balance. Colección Popular 676, Fondo de Cultura Económica. 
Devalle De Rendo, Alicia. (1995). La capacitación docente. Editorial Magisterio del Río de la Plata.

Granados, H., \& García, C. (2016). El modelo de aprendizaje experiencial como alternativa para mejorar el proceso de aprendizaje en el aula. Ánfora, 37-54.

Godino, Juan. D. (2000). La consolidación de la educación Matemática como disciplina científica. Las

Matemáticas del siglo XX. Una mirada en 101 artículos.

Haydee, P. (2007). P.E.R (Preguntar, Escuchar, Responder) Una Triada Para Enseñar, Comprender Y

Aprender Historia, N. 28. Vol.Xv

Luis, T. E. (2013). Defendiendo lo colectivo: combatir el conformismo, promover la educcaión pública. Revista Interuniversitaria de Formación del Profesorado, 115-123.

Marisela, S. (2007). Estrategias docentes y capacidades metacognitivas del alumno en el área de lengua y literatura en educación básica, Universidad Nacional Abierta UNA.

Ortiz. O, Alexander. (2005). Centro de Estudios Pedagógicos y Didácticos CEPEDID.

Patricia, Z. M. (2008). Actas del II Congreso de Innovación Docente en Ciencias Jurídicas: Hacia el Espacio Europeo de Educación Superior, Metodología de la enseñanza de la Historia del Derecho ante el Espacio Europeo de Educación Superior

Rafael, P. (1993). Contructivismo y escuela. Sevilla: Díada Editora, S.L. Obtenido de Hacia un modelo de enseñanza-aprendizaje basado en la investigación.
Tierno, B. (2003). Las mejores técnicas de estudio. Temas de Hoy. 